\title{
ON TABACHNIKOV'S CONJECTURE
}

\author{
A. I. NAZAROV AND F. V. PETROV
}

To V. A. Zalgaller on the occasion of his 85th birthday with love and great respect

Abstract. Tabachnikov's conjecture is proved: for any closed curve $\Gamma$ lying inside a convex closed curve $\Gamma_{1}$ the mean absolute curvature $T(\Gamma)$ exceeds $T\left(\Gamma_{1}\right)$ if $\Gamma \neq k \Gamma_{1}$.

\section{$\S 1$. The PROBLEM SETting AND MAIN IDEAS}

Let $\Gamma(s), s \in[0, L(\Gamma)]$, be a naturally parametrized closed curve on a plane. We say that $\Gamma(s)$ belongs to the class $B V^{1}$ if the velocity $\Gamma^{\prime}(s)$ exists and is continuous everywhere on $[0, L(\Gamma)]$ except for a countable set; at the points of this set $\Gamma^{\prime}$ has left and right limits, and the variation of $\Gamma^{\prime}$ is bounded. ${ }^{1}$ The full variation of $\Gamma^{\prime}$ is called the full rotation of the curve $\Gamma$ and is denoted by $V(\Gamma)$.

The full rotation has the following properties.

$1^{\circ}$. For $C^{2}$-smooth curves, the full rotation is equal to the integral of the absolute value of the curvature with respect to arc length.

$2^{\circ}$. The full rotation of a closed polygonal line equals the sum of the external angles at all of its vertices.

$3^{\circ}$. The full rotation of a closed convex curve exists and is equal to $2 \pi$.

We define the mean absolute curvature of a curve $\Gamma \in B V^{1}$ as its full rotation divided by its length: $T(\Gamma)=V(\Gamma) / L(\Gamma)$.

In [1], S. L. Tabachnikov formulated the following conjecture, which he called the DNA inequality.

Theorem $\mathcal{P}$. 1. The mean absolute curvature $T(\Gamma)$ of a closed curve $\Gamma \in B V^{1}$ ("DNA") lying inside a closed convex curve $\Gamma_{1}$ ("cell") does not exceed $T\left(\Gamma_{1}\right)$.

2. If $T(\Gamma)=T\left(\Gamma_{1}\right)$, then $\Gamma$ is a multiple circuit of $\Gamma_{1}$.

A survey of results concerning this conjecture and its generalisations was given in [1]. The first part of Theorem $\mathcal{P}$ was proved in [2].

We prove the DNA inequality in full generality. The proof of the first part partially follows the strategy of [2], but is clearer and is used in the proof of the second part. In order to make the paper self-contained, we give (significantly simpler) proofs of all lemmas from [2] that we use.

Without loss of generality we may assume that $\Gamma_{1}$ is the boundary of the convex hull of $\Gamma$.

2000 Mathematics Subject Classification. Primary 53A04; Secondary 52A40, 52A10.

Key words and phrases. Geometric inequalities, mean absolute curvature, convexity.

Supported by grant NSh-8336.2006.1 (the first author) and by grants NSh-2251.2003.1 and RFFR 05-01-00899 (the second author).

${ }^{1}$ The variation of a function $f$ with values in the unit circle is defined as the supremum of the sums $\sum_{i=1}^{n} \rho\left(f\left(t_{i}\right), f\left(t_{i-1}\right)\right)+\rho\left(f\left(t_{n}\right), f\left(t_{0}\right)\right)$. Here $t_{0}<t_{1}<\cdots<t_{n}$ is any partition of the segment that is the domain of $f$ with the property that $f$ is defined at the nodes $t_{i}$, and $\rho$ is the intrinsic metric of the circle. 
A curve $\widetilde{\Gamma}$ is said to be better than a curve $\Gamma$ if $T(\Gamma) \geq T(\widetilde{\Gamma})$, and $\widetilde{\Gamma}$ is strictly better if $T(\Gamma)>T(\widetilde{\Gamma})$. By (strict) improvement of a curve we mean replacement of this curve with a (strictly) better one, provided the convex hull of the "new" curve is contained in the convex hull of the "old" one. If after some improvements of a curve $\Gamma$ we obtain a multiple circuit of $\Gamma_{1}$, then Claim 1 of Theorem $\mathcal{P}$ is proved for $\Gamma$. If, moreover, at least one improvement is strict, then the strict inequality $T(\Gamma)>T\left(\Gamma_{1}\right)$ is established.

First, we reduce Claim 1 to the case of polygonal lines. After that, the vertices of a polygonal line are moved to the boundary (here and below: the boundary of the convex hull). Now each change in the turn direction admits an improvement of the curve. After a finite number of such improvements, we arrive at a curve that rotates in only one direction (say, only clockwise). For these curves Claim 1 is almost obvious. Then we prove that if a curve differs from the multiple circuit of the boundary, it can be improved strictly. This proves Claim 2.

\section{§2. Reduction to A POlygonal Line}

Consider a point of jump of $\Gamma^{\prime}$. Note that the variation of $\Gamma^{\prime}$ will not change if we redefine $\Gamma^{\prime}$ at this point to be an arbitrary vector lying on the unit circle between ${ }^{2}$ the left and the right limit of $\Gamma^{\prime}$. Next, talking about the set of velocity values on some subinterval, we shall mean that we add to it the above-mentioned sets of admissible values at jump points. So, the set of velocity values on any interval is an arc of the unit circle.

Lemma 1. Consider two points $A$ and $B$ on a curve $\Gamma$. Suppose that $\Gamma^{\prime}(A)$ and $\Gamma^{\prime}(B)$ exist. Then the full rotation of $\Gamma$ between $A$ and $B$ (this part of $\Gamma$ will be denoted by $\left.\Gamma_{A B}\right)$ is not less than $\rho\left(\Gamma^{\prime}(A), e\right)+\rho\left(\Gamma^{\prime}(B), e\right)$, where $e$ is the unit vector directed as $\overline{A B}$.

Proof. If the vector $e$ lies in the range of $\Gamma^{\prime}$ on the part from $A$ to $B$, then the claim is trivial (it suffices to consider a partition of $\Gamma_{A B}$ with nodes $A$ and $B$, and a point $C$ with $\Gamma^{\prime}(C)=e$ ). If not, the set of values of $\Gamma^{\prime}$ from $A$ to $B$ is an arc including a semicircle (otherwise one could find a half-plane that contains this set and does not contain $\overline{A B}$ ). Consider a sufficiently fine partition of $\Gamma_{A B}$. We see that the rotation of $\Gamma$ from $A$ to $B$ cannot be less than the larger arc between $\Gamma^{\prime}(A)$ and $\Gamma^{\prime}(B)$. This proves the lemma in this case.

Lemma 2. Assume that Claim 1 of Theorem $\mathcal{P}$ fails for a curve $\Gamma$. Then there exists a polygonal line for which this claim also fails.

Proof. By assumption, we have $T(\Gamma)<T\left(\Gamma_{1}\right)$. We inscribe a polygonal line $\Delta$ into $\Gamma$ so that the length of $\Delta$ is close to the length of $\Gamma$ (namely, we require $\left.L(\Delta)>L(\Gamma) \cdot \frac{T(\Gamma)}{T\left(\Gamma_{1}\right)}\right)$. Clearly, the convex hull of $\Delta$ (we denote by $\Delta_{1}$ its boundary) lies inside $\Gamma_{1}$. We may also assume that $\Gamma^{\prime}$ is defined at all vertices of $\Delta$.

To prove that $V(\Delta) \leq V(\Gamma)$, it suffices to sum up the inequalities of Lemma 1 over all edges of a polygonal line, and then use the triangle inequality.

Hence,

$$
T(\Delta)=\frac{V(\Delta)}{L(\Delta)}<\frac{V(\Gamma)}{L(\Gamma)} \cdot \frac{T\left(\Gamma_{1}\right)}{T(\Gamma)}=T\left(\Gamma_{1}\right) \leq T\left(\Delta_{1}\right),
$$

and the lemma follows.

Let $A_{1} A_{2} \ldots A_{n} A_{1}$ be a closed polygonal line. We denote by $L$ its length, by $P$ the perimeter of its convex hull, and by $V:=\sum_{i=1}^{n}\left(\pi-\angle A_{i-1} A_{i} A_{i+1}\right)$ its full rotation (the enumeration is cyclic modulo $n$ ). We assume that no vertex $A_{i}$ of a polygonal line lies

\footnotetext{
${ }^{2}$ That is, on the smaller of the two arcs.
} 
on the segment $\left[A_{i-1} A_{i+1}\right]$. Such vertices can appear in the process of improvement, and then they will be removed immediately.

In the above notation, Claim 1 of Theorem $\mathcal{P}$ for polygonal lines can be reformulated as follows.

Lemma 3. $\frac{L}{V} \leq \frac{P}{2 \pi}$.

Observe that Lemmas 2 and 3 imply Claim 1 in the general case.

\section{§3. Quadrilaterals}

Here we prove two lemmas, which give the claim of Lemma 3 for quadrilaterals. These lemmas will be used in what follows for improvement of an arbitrary polygonal line.

Lemma 4. For any triangle $A B C$ we have

$$
\frac{A B+B C}{2 \pi-\beta}<\frac{A B+B C+A C}{2 \pi}
$$

with $\beta=\angle A B C$.

Proof. By the sine theorem,

$$
\frac{A B+B C}{A C}=\frac{\sin \angle A+\sin \angle C}{\sin \beta}=\frac{2 \sin \left(\frac{\angle A+\angle C}{2}\right) \cos \left(\frac{\angle A-\angle C}{2}\right)}{2 \sin \frac{\beta}{2} \cos \frac{\beta}{2}}=\frac{\cos \left(\frac{\angle A-\angle C}{2}\right)}{\sin \frac{\beta}{2}} \leq \frac{1}{\sin \frac{\beta}{2}} .
$$

The concavity of the sine function on $[0, \pi / 2]$ implies that

$$
\sin \frac{\beta}{2}>\frac{\beta}{\pi} \text {. }
$$

Consequently,

$$
\frac{A B+B C}{A C}<\frac{\pi}{\beta}<\frac{2 \pi-\beta}{\beta}
$$

which is equivalent to (1).

Lemma 5. Let $A B C D$ be a convex quadrilateral, and let $O$ be the intersection point of its diagonals. Put $\varphi=\angle A O B$. Then

$$
\frac{A B+B D+D C+C A}{2(\pi+\varphi)}<\frac{A B+B C+C D+D A}{2 \pi} .
$$

Proof. By Lemma 4, we have $A B>\frac{\varphi}{\pi}(A O+O B), C D>\frac{\varphi}{\pi}(C O+O D)$. Adding these inequalities, we get

Similarly,

$$
A B+C D>\frac{\varphi}{\pi}(A C+B D)
$$

Hence,

$$
B C+A D>\left(1-\frac{\varphi}{\pi}\right) \cdot(A C+B D)
$$

$$
\frac{\varphi}{\pi} \cdot \frac{A B+C D}{A C+B D}+\left(1+\frac{\varphi}{\pi}\right) \cdot \frac{B C+D A}{A C+B D}>\frac{\varphi}{\pi} \cdot \frac{\varphi}{\pi}+\left(1+\frac{\varphi}{\pi}\right) \cdot\left(1-\frac{\varphi}{\pi}\right)=1 .
$$

Therefore,

$$
\frac{A B+C D}{A C+B D}+1<\left(1+\frac{\varphi}{\pi}\right) \cdot\left(\frac{A B+C D}{A C+B D}+\frac{B C+D A}{A C+B D}\right) .
$$

This inequality is equivalent to (2).

The statements of Lemmas 4 and 5 are none other than the particular cases of Lemma 3 for concave and self-intersecting quadrilaterals, respectively. 
Remark 1. Inequalities (1) and $(2)($ with $\leq)$ are valid also for a degenerate triangle $A B C$ and quadrilateral $A B C D$, respectively.

\section{§4. Moving Vertices to the boundary}

Here we reduce the proof of Lemma 3 to the case where all vertices of a polygonal line belong to the boundary of its convex hull.

Let a vertex $A_{i}$ be situated strictly inside the convex hull. We consider three cases.

Case a). The line $A_{i} A_{i+1}$ does not separate the points $A_{i-1}$ and $A_{i+2}$. In this case we can strictly improve a polygonal line, increasing its length without changing the rotation: we move the vertex $A_{i}$ beyond the segment $A_{i-1} A_{i}$ while it touches either the boundary or the ray $A_{i+2} A_{i+1}$. In this way, we get a better polygonal line with a smaller number of vertices situated strictly inside the convex hull (it may well happen that the total number of vertices will also reduce). A similar operation is possible if $A_{i} A_{i-1}$ does not separate $A_{i+1}$ and $A_{i-2}$.

Case b). Assume that the line $A_{i} A_{i+1}$ separates $A_{i-1}$ and $A_{i+2}$, while $A_{i} A_{i-1}$ separates $A_{i+1}$ and $A_{i-2}$. Let $A_{i-2}$ and $A_{i+2}$ be situated in the angles supplementary (with respect to the side containing $\left.A_{i}\right)$ to the angles $\angle A_{i+1} A_{i-1} A_{i}$ and $\angle A_{i-1} A_{i+1} A_{i}$, respectively. Then we replace the edges $A_{i-1} A_{i}$ and $A_{i} A_{i+1}$ of the polygonal line $A_{1} A_{2} \ldots A_{n}$ with one edge $A_{i-1} A_{i+1}$.

Assume that the initial polygonal line does not satisfy the inequality of Lemma 3, while the new one does; i.e.,

$$
\frac{L}{V}>\frac{P}{2 \pi} \geq \frac{L-\left(A_{i-1} A_{i}+A_{i} A_{i+1}-A_{i-1} A_{i+1}\right)}{V-2(\pi-\beta)},
$$

with $\beta=\angle A_{i-1} A_{i} A_{i+1}$. Then

$$
P \cdot V-2(\pi-\beta) \cdot P+2 \pi\left(A_{i-1} A_{i}+A_{i} A_{i+1}-A_{i-1} A_{i+1}\right) \geq 2 \pi L>P \cdot V,
$$

whence

$$
\begin{aligned}
2 \pi\left(A_{i-1} A_{i}+A_{i} A_{i+1}\right. & \left.-A_{i-1} A_{i+1}\right) \\
& >2(\pi-\beta) P \geq 2(\pi-\beta)\left(A_{i-1} A_{i}+A_{i} A_{i+1}+A_{i-1} A_{i+1}\right),
\end{aligned}
$$

in contradiction with Lemma 4 . Thus, the new polygonal line must also be a counterexample to Lemma 3, but with a smaller number of inner vertices.

It remains to consider case c) where, for example, the line $A_{i-1} A_{i+1}$ separates $A_{i+2}$ and $A_{i}$ (in this case $A_{i+1}$ also lies strictly inside the convex hull). Without loss of generality we may assume that the angle $A_{i-1} A_{i+1} A_{i}$ is the smallest for all indices $i$ satisfying this condition. We replace $i$ by $i+1$ and consider similar cases. The vertex $A_{i-1}$ lies in the angle supplementary to $\angle A_{i} A_{i+2} A_{i+1}$ with respect to $A_{i} A_{i+1}$. If the vertex $A_{i+3}$ does not lie in the angle vertical to $A_{i+1} A_{i+2} A_{i}$, the polygonal line can be improved as was shown (with the change $i \rightarrow i+1$ ). Otherwise, we get a contradiction with our choice of $i$ : the angle $\angle A_{i} A_{i+2} A_{i+1}$ is less than the angle $\angle A_{i} A_{i+1} A_{i-1}$ (because $\left.\angle A_{i} A_{i+2} A_{i+1}+\angle A_{i} A_{i+1} A_{i+2}<\angle A_{i} A_{i+1} A_{i_{1}}+\angle A_{i} A_{i+1} A_{i+2}\right)$.

So, after finitely many steps, we reduce the general case to the case where all the vertices $A_{i}$ of a polygonal line $A_{1} A_{2} \ldots A_{n}$ lie on the boundary of its convex hull.

\section{§5. REDUCING THE NUMBER OF DIRECTION CHANGES}

We fix an orientation of the plane. We say that a polygonal line $A_{1} A_{2} \ldots A_{n}$ turns to the right at the vertex $A_{i}$ if the basis $\overline{A_{i-1} A_{i}}, \overline{A_{i} A_{i+1}}$ is negatively oriented. Otherwise (in particular, if these vectors are collinear) we say that it turns to the left.

If the polygonal line turns to the right (or to the left) two times in succession, we can replace the edge between these turns by the part of the boundary passed in the same 
direction. This operation improves a polygonal line. We call it stretching of a polygonal line.

Lemma 6. Assume that some consequent edges of our polygonal line form a full circuit of the boundary, and that the first and the last edges coincide (i.e., the boundary is a convex polygon $C_{1} C_{2} \ldots C_{m}$ and the polygonal line has a part $\left.X C_{1} C_{2} \ldots C_{m} C_{1} C_{2} Y\right)$. Then the claim of Lemma 3 for this polygonal line is equivalent to the claim of Lemma 3 for the polygonal line obtained by removing this part (i.e., for the polygonal line in which this part is replaced with $\left.X C_{1} C_{2} Y\right)$.

Proof. Observe that the perimeter of the polygonal line obtained after removing the circuit is equal to $L-P$, and its full rotation is $V-2 \pi$. For the new polygonal line, Lemma 3 states that $\frac{L-P}{V-2 \pi} \leq \frac{P}{2 \pi}$, which is equivalent to the statement of Lemma 3 for the initial polygonal line.

Let us repeat the operation of Lemma 6 while this is possible. This process must stop because the number of edges decreases. We observe that the number of changes in the turn direction will remain intact.

Now the polygonal line is split into parts in which all the turns have the same direction; moreover, in each part all the edges (except, possibly, the first and the last) go along the boundary and, by Lemma 6 , are distinct.

We shall do the following operation. We choose a part $A_{i} A_{i+1} \ldots A_{k}$ in which all turns are, say, left (more precisely, the turns at the vertices $A_{i+1}, A_{i+2}, \ldots, A_{k-1}$ are left, and the turns at $A_{i}$ and $A_{k}$ are right). We replace the path $A_{i} A_{i+1} \ldots A_{k}$ by the part of the boundary $A_{i} \ldots A_{k}$ going along the boundary in the opposite direction (in our case, this is the negative direction). The number of turn-direction changes decreases after this operation.

Assume that the initial polygonal line does not satisfy the inequality of Lemma 3 . Our goal is to prove that in this case the new polygonal line also does not. Six cases are possible, determined by the order of the vertices $A_{i}, A_{i+1}, A_{k-1}, A_{k}$ while going along the boundary in the positive direction:

$$
\begin{aligned}
& 1^{\circ} \cdot A_{i} A_{i+1} A_{k-1} A_{k} ; \\
& 2^{\circ} \cdot A_{i} A_{k} A_{i+1} A_{k-1} ; \\
& 3^{\circ} \cdot A_{i} A_{i+1} A_{k} A_{k-1} ; \\
& 4^{\circ} . A_{i} A_{k-1} A_{k} A_{i+1} ; 5^{\circ} . A_{i} A_{k-1} A_{i+1} A_{k} ; \\
& 6^{\circ} . A_{i} A_{k-1} A_{k} A_{i+1} .
\end{aligned}
$$

Cases 3 and 4 are equivalent up to renaming the vertices and symmetry. We denote the length of the polygonal line $A_{i} A_{i+1} \ldots A_{k}$ by $s$ and the length of its replacement by $s^{\prime}$.

$1^{\circ}$ (see Figure 1). Let $\angle A_{i+1} A_{i} A_{k}=\alpha, \angle A_{k-1} A_{k} A_{i}=\beta$. After the change of the polygonal line, its full rotation reduces by $2(\alpha+\beta)$, and the length reduces by $s-s^{\prime}$.

If the new polygonal line satisfies the inequality of Lemma 3 , then

$$
\frac{L}{V}>\frac{P}{2 \pi} \geq \frac{L+s^{\prime}-s}{V-2(\alpha+\beta)}
$$

or

$$
P \cdot V-2 P(\alpha+\beta)+2 \pi\left(s-s^{\prime}\right) \geq 2 \pi L>P \cdot V,
$$

whence $2 \pi\left(s-s^{\prime}\right)>2(\alpha+\beta) P \geq 2(\alpha+\beta)\left(s+s^{\prime}\right)$, and $2(\pi-\alpha-\beta) s>2(\pi+\alpha+\beta) s^{\prime}$.

The latter inequality can be valid only if $\alpha+\beta<\pi$. In this case the rays $A_{i} A_{i+1}$ and $A_{k} A_{k-1}$ meet at a point $C$, and $C A_{i}+C A_{k} \geq s, A_{i} A_{k} \leq s^{\prime}$. So,

$$
(\pi-\alpha-\beta)\left(C A_{i}+C A_{k}\right)>(\pi+\alpha+\beta) A_{i} A_{k},
$$

which contradicts Lemma 4. 


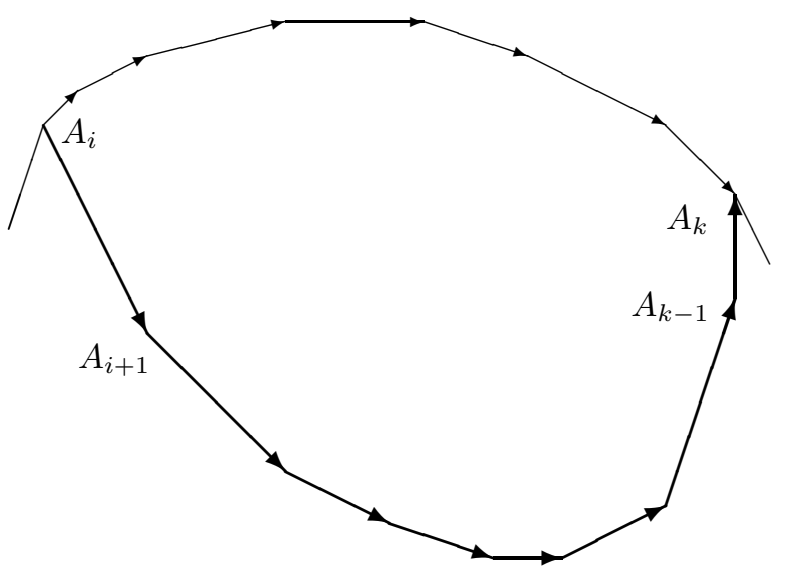

Figure 1

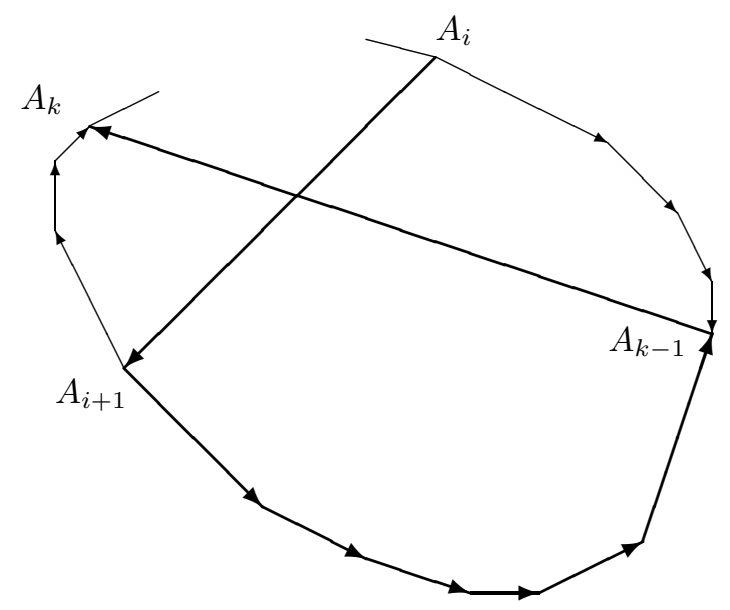

Figure 2

$2^{\circ}$ (see Figure 2). Let $O$ denote the point of intersection of the segments $A_{i} A_{i+1}$ and $A_{k} A_{k-1}, \angle A_{i} O A_{k}=\varphi$. After the change of the polygonal line, its full rotation reduces by $2 \varphi$, and the length reduces by $s-s^{\prime}$.

If the new polygonal line satisfies Lemma 3, then

$$
\frac{L}{V}>\frac{P}{2 \pi} \geq \frac{L+s^{\prime}-s}{V-2 \varphi} .
$$

Arguing as in $1^{\circ}$, we deduce that $2 \pi\left(s-s^{\prime}\right)>2 \varphi P$, whence

$$
\frac{A_{i} A_{i+1}+A_{k} A_{k-1}-\left(A_{i} A_{k-1}+A_{k} A_{i+1}\right)}{A_{i} A_{k}+A_{k} A_{i+1}+A_{i+1} A_{k-1}+A_{i} A_{k-1}}>\frac{\varphi}{\pi} .
$$

This contradicts Lemma 5 (for the quadrilateral $A_{i} A_{k-1} A_{i+1} A_{k}$ ).

The other cases are analyzed much as $1^{\circ}$ and $2^{\circ}$; Lemma 4 is used in cases $3^{\circ}$ and $6^{\circ}$, Lemma 5 is used in case $5^{\circ}$.

So, after a finite number of steps we get a polygonal line that turns only to the right. Using stretching, we get a multiple circuit of a boundary; hence, the statement of Lemma 
3 is valid for this polygonal line. Thus, the initial assumption was wrong, and Lemma 3 is proved. Claim 1 of Theorem $\mathcal{P}$ is also proved.

\section{$\S 6$. Proof of Claim 2}

Assume that the curve $\Gamma$ is not a (multiple) boundary circuit, but $T(\Gamma)=T\left(\Gamma_{1}\right)$. We select finitely many points on $\Gamma$ so that the sum of the velocity jumps at the other points is small (say, less than $\pi / 180$ ). The union of this finite set and the set $\Gamma \cap \Gamma_{1}$ is closed. The preimage (recall that the curve is naturally parametrized) of its complement is a union of a countable collection of intervals. Consider one of these intervals; let it correspond to the part of $\Gamma$ between $A$ and $B$.

A part $\Gamma_{C D}$ is said to be small if the set of velocity values on this part is an arc of length at most $\pi / 4$, and the circle with diameter $C D$ lies strictly inside $\Gamma_{1}$. It is easily seen that any inner point of $\Gamma_{A B}$ belongs to some small subpart.

Consider a small part $\Gamma_{C D}$. We redefine, if necessary, the velocities $\Gamma^{\prime}(C)$ and $\Gamma^{\prime}(D)$ to be their right limits. Define a parallelogram $C P D Q$ with vectors $\overline{C P}$ and $\overline{C Q}$ directed as the extremal directions of the velocity of $\Gamma$ on the part $\Gamma_{C D}$. This parallelogram lies strictly inside $\Gamma_{1}$ (because the extremal directions are close to the direction of the vector $\overline{C D})$.

There are points $X$ and $Y$ on $\Gamma_{C D}$ with tangents parallel to $C P$ and $C Q$, respectively. Without loss of generality we may assume that the order of the points is $C-X-Y-D$. We replace the part $\Gamma_{C D}$ with the polygonal line $C P D$.

Note that the full rotation of $\Gamma_{C D}$ is not less than

$$
v:=\rho\left(\Gamma^{\prime}(C), \Gamma^{\prime}(X)\right)+\rho\left(\Gamma^{\prime}(X), \Gamma^{\prime}(Y)\right)+\rho\left(\Gamma^{\prime}(Y), \Gamma^{\prime}(D)\right),
$$

while the full rotation of the new part equals $v$. Equality occurs only if $\Gamma$ is convex from $C$ to $X$, from $X$ to $Y$, and from $Y$ to $D$.

Furthermore, the length of $\Gamma_{C D}$ does not exceed $C P+P D$. To prove this, consider an arbitrary polygonal line inscribed in $\Gamma_{C D}$. The directions of its edges can range between the directions of $\overline{C P}$ and $\overline{C Q}$. Therefore, after monotone rearrangement of them (in the sense of direction) from $\overline{C P}$ to $\overline{C Q}$, we get a convex polygonal line $C \ldots D$ situated inside the triangle $C P D$, so that its length does not exceed $C P+P D$.

So, after replacement of $\Gamma_{C D}$ by the polygonal line $C P D$, the full rotation does not decrease, and the length does not increase; i.e., the curve $\Gamma$ improves. But it cannot be strictly improved, due to Claim 1 already proved. Hence neither full rotation nor length changes after such a replacement. The first is possible only if $\Gamma_{C D}$ splits into at most three convex parts $(C-X, X-Y, Y-D)$. The second is possible only if each of these parts is a polygonal line with at most two edges. Thus, $\Gamma_{C D}$ is a polygonal line with at most six edges (the number of edges can be reduced, but we do not need this now).

We fix points $A^{\prime}$ and $B^{\prime}$ on the open $\operatorname{arc} \Gamma_{A B}$ and cover $\Gamma_{A^{\prime} B^{\prime}}$ by finitely many small parts. The curve $\Gamma$ is a polygonal line on each small part; hence $\Gamma_{A^{\prime} B^{\prime}}$ is also a polygonal line.

Now we prove that if $\Gamma_{A^{\prime} B^{\prime}}$ has at least four edges, then $\Gamma$ can be strictly improved. Consider the cases of $\S 4$. In case a) only the local structure of a polygonal line was used, and the same argument works in our situation. In case b), using Claim 1 for the modified curve, we obtain an inequality similar to (3):

$$
\frac{L(\Gamma)}{V(\Gamma)}=\frac{L\left(\Gamma_{1}\right)}{2 \pi} \geq \frac{L(\Gamma)-\left(A_{i-1} A_{i}+A_{i} A_{i+1}-A_{i-1} A_{i+1}\right)}{V(\Gamma)-2(\pi-\beta)},
$$

whence

$$
2 \pi\left(A_{i-1} A_{i}+A_{i} A_{i+1}-A_{i-1} A_{i+1}\right) \geq 2(\pi-\beta)\left(A_{i-1} A_{i}+A_{i} A_{i+1}+A_{i-1} A_{i+1}\right),
$$

which contradicts Lemma 4. 
In case c), if $A_{i+2}$ and $A_{i}$ are separated by the line $A_{i-1} A_{i+1}$ (then the vertex $A_{i+1}$ also lies strictly inside the convex hull), the polygonal line can be strictly improved by replacing the edge $A_{i} A_{i+1}$ with a parallel longer edge $A_{i}^{\prime} A_{i+1}^{\prime} \| A_{i} A_{i+1}$, where $A_{i} \in$ $\left[A_{i-1} A_{i}^{\prime}\left[, A_{i+1}^{\prime} \in\right] A_{i+1} A_{i+2}\right]$.

Thus, $\Gamma_{A^{\prime} B^{\prime}}$ is a polygonal line with at most three edges. Since $A^{\prime}$ and $B^{\prime}$ were chosen arbitrarily, the curve $\Gamma_{A B}$ is a polygonal line with at most three edges.

Now we add the points of "large turn", excluded before, to the intervals under consideration. Then the entire inner part of $\Gamma$ is split into an at most countable set of parts; every part is a polygonal line with a finite number of edges.

If a part contains more than one edge, it contains inner vertices, and the curve $\Gamma$ can be strictly improved as in $\S 4$.

So, all points of $\Gamma$ lie either on the boundary, or on a segment joining boundary points.

Suppose that the number of segments is infinite. Then there exists a sequence of segments with length tending to zero and endpoints tending to some point $C \in \Gamma_{1}$. We fix a small neighborhood of $C$ where the full rotation of $\Gamma_{1}$ is equal to $\varphi_{0}<\pi$. Consider one of the segments $\overline{A B}\left(A, B \in \Gamma_{1}\right)$ lying in this neighborhood. If the vectors $\Gamma^{\prime}(A-)$ and $\Gamma^{\prime}(B+)$ look to different half-planes with respect to the line $A B$, then the curve $\Gamma$ can be strictly improved by stretching the segment $A B$ to the boundary. Since this is impossible, the variation of $\Gamma^{\prime}$ on $A B$ is at least $\pi-\varphi_{0}$, so that the full variation is infinite, a contradiction.

So, the curve $\Gamma$ consists of finitely many boundary pieces and finitely many segments joining them. If $\Gamma$ contains return points on the boundary (since $\Gamma \in B V^{1}$, there can be only a finite number of such points), we view them as "inner segments of zero length".

If two consecutive pieces of the boundary have the same circuit direction, we can strictly improve $\Gamma$ by stretching the segment between them to the boundary. Also we can remove all the full circuits of the boundary as in Lemma 6 .

Now, consider an arc $\Gamma_{A B}$ consisting of the segment $A A_{1}$, the piece of the boundary $\Gamma_{A_{1} B_{1}}$ (which has, say, positive direction), and the segment $B_{1} B$. As in $\S 5$, we replace $\Gamma_{A B}$ with a "negative" arc of the boundary between $A$ and $B$. Here we must analyze the six cases of $\S 5$ again, depending on the order of the points $A, A_{1}, B, B_{1}$ in a positive circuit. For example, in case $1^{\circ}$ (the order $A A_{1} B_{1} B$ ), we use Claim 1 for the modified curve to get an inequality similar to (4):

$$
\frac{L(\Gamma)}{V(\Gamma)}=\frac{L\left(\Gamma_{1}\right)}{2 \pi} \geq \frac{L(\Gamma)+s^{\prime}-s}{V(\Gamma)-2\left(\angle A_{1} A B+\angle B_{1} B A\right)} .
$$

As in $\S 5$, this implies that the rays $A A_{1}$ and $B B_{1}$ meet at a point $C$ with

$$
\left(\pi-\angle A_{1} A B-\angle B_{1} B A\right) \cdot(C A+C B) \geq\left(\pi+\angle A_{1} A B+\angle B_{1} B A\right) \cdot A B,
$$

which contradicts Lemma 4.

Similar contradictions can be obtained in the remaining cases. This shows that the curve $\Gamma$ cannot have inner segments; hence, it is a circuit of the boundary. Taking the circuits removed earlier into account, we see that the initial curve $\Gamma$ was a multiple circuit of a boundary. Claim 2 is proved.

\section{$\S 7$. Surfaces of COnstant CuRvature}

In this section we prove an analog of the DNA inequality for the sphere.

Let $\Gamma$ be a closed curve lying in some hemisphere (here and in what follows: of unit radius). Suppose that the variation of the right rotation $V(\Gamma)$ is finite. For the definitions we refer the reader to [3]. Note that if $\Gamma: A_{1} A_{2} \ldots A_{n} A_{1}$ is a closed polygonal line, then $V(\Gamma)=\sum_{i=1}^{n}\left(\pi-\angle A_{i-1} A_{i} A_{i+1}\right)$ (enumeration of indices is cyclic). If $\Gamma$ is a $C^{2}$-smooth 
closed curve, then $V(\Gamma)$ is equal to the integral of the absolute value of the geodesic curvature vector with respect to the natural parameter.

We define the mean absolute geodesic curvature $T(\Gamma)$ of a closed curve in a hemisphere as

$$
T(\Gamma):=V(\Gamma) / L(\Gamma)
$$

Theorem $\mathcal{S}$. Let $\Gamma$ be a closed curve in a hemisphere, and let the variation of its right rotation be finite. Let $\Gamma_{1}$ be the boundary of its convex hull. Then $T(\Gamma) \geq T\left(\Gamma_{1}\right)=$ $(2 \pi-S) / L\left(\Gamma_{1}\right)$, where $S$ is the area of the convex hull.

The plan of the proof of Theorem $\mathcal{S}$ is the same as in the planar case. First, we formulate the corresponding statement for polygonal lines.

Theorem $\mathcal{S}^{\prime}$. Let $\Gamma$ be a closed polygonal line in a hemisphere, and let $\Gamma_{1}$ be the boundary of its convex hull. If $\Gamma$ is not a multiple circuit of $\Gamma_{1}$, then $T(\Gamma)>T\left(\Gamma_{1}\right)$.

Before we pass to the case of quadrilaterals, we prove the following claim, which refines (in a particular case) the A. D. Aleksandrov theorem on comparing angles.

Lemma $1 s$. Let $A B C$ be a nondegenerate triangle on a sphere. Denote its sides by $B C=a, C A=b, A B=c$, and its angles by $\alpha, \beta, \gamma$, respectively. Let $\alpha^{\prime}, \beta^{\prime}, \gamma^{\prime}$ be the angles of a triangle with sides $a, b, c$ on a plane. Then

$$
\alpha-\alpha^{\prime}<\left(\beta-\beta^{\prime}\right)+\left(\gamma-\gamma^{\prime}\right) \text {. }
$$

Proof. We denote $a+b+c=4 S, S-a / 2=X, S-b / 2=Y, S-c / 2=Z$. Let $\mathcal{E}=\alpha+\beta+\gamma-\pi$ be the area of the triangle $A B C$. Inequality $\left(1_{s}\right)$ is equivalent to the inequality $\alpha^{\prime}>\alpha-\mathcal{E} / 2$ or

$$
\tan \frac{\alpha^{\prime}}{2}>\tan (\alpha / 2-\mathcal{E} / 4)
$$

Substituting the formulas

$$
\begin{aligned}
\tan \frac{\alpha}{2} & =\sqrt{\frac{\sin 2 Y \sin 2 Z}{\sin 2 X \sin 2 S}}, \\
\tan \frac{\alpha^{\prime}}{2} & =\sqrt{\frac{Y Z}{X S}}, \\
\tan \frac{\mathcal{E}}{4} & =\sqrt{\tan S \cdot \tan X \cdot \tan Y \cdot \tan Z}
\end{aligned}
$$

(the first formula is $[4,(28)]$, the second is $[5,(20)]$, the third is $[6])$, we convert $\left(2_{s}\right)$ into the inequality

$$
\frac{\sin Z \sin Y}{\sin X \sin S} \cdot \frac{\cos Y \cos Z-\sin X \sin S}{\cos X \cos S+\sin Y \sin Z}<\sqrt{\frac{Y Z}{X S}} \cdot \sqrt{\frac{\sin 2 Z \sin 2 Y}{\sin 2 X \sin 2 S}} .
$$

Since $S=X+Y+Z$, we have $\cos (S-X)=\cos (Y+Z)$; hence, the second factor on the left in $\left(3_{s}\right)$ equals 1 . Denoting $f(x)=x \cot x$, we reduce $\left(3_{s}\right)$ to

$$
f(Y) f(Z)>f(X) f(X+Y+Z) .
$$

Since $f^{\prime}(x)=\frac{\sin (2 x)-2 x}{2 \sin ^{2} x}<0$ for $0<x<\frac{\pi}{2}$, the function $f$ is strictly monotone decreasing on $\left[0, \frac{\pi}{2}\right]$. Since all the arguments in $\left(4_{s}\right)$ lie in $\left[0, \frac{\pi}{2}\right]$ (we recall that $X+Y+Z=$ $(a+b+c) / 4 \leq \pi / 2)$, we may assume that $X=0$ and prove the inequality

$$
f(Y) f(Z)>f(0) f(Y+Z) .
$$

We have $(\ln (f))^{\prime \prime}(x)=\frac{4}{\sin ^{2}(2 x)}\left(\cos (2 x)-\frac{\sin ^{2}(2 x)}{4 x^{2}}\right)$. We omit an elementary proof of the inequality $\cos t<\left(\frac{\sin t}{t}\right)^{2}$ for $\left.t=2 x \in\right] 0, \pi[$. This shows that $\ln (f)$ is strictly concave on $\left[0, \frac{\pi}{2}\right]$, and $\left(5_{s}\right)$ follows. 
Now we are ready to prove analogs of Lemmas 4 and 5 for the sphere.

Lemma $2 s$. Let $A B C$ be a nondegenerate triangle on the sphere. Then, with the same notation as in Lemma 1 , we have

$$
\frac{a+c}{2 \pi-\beta}<\frac{a+b+c}{2 \pi-\mathcal{E}}
$$

Proof. This follows from the chain of inequalities

$$
\frac{a+c}{a+b+c}<\frac{2 \pi-\beta^{\prime}}{2 \pi}<\frac{2 \pi-\beta+\mathcal{E} / 2}{2 \pi} \leq \frac{2 \pi-\beta}{2 \pi-\mathcal{E}}
$$

(the first inequality is Lemma 4 , the second is Lemma $1 s$, and the third reduces to the obvious $\beta \leq \pi+\mathcal{E} / 2)$.

Lemma 3s. For a convex spherical quadrilateral $A B C D$ on a hemisphere, let $O$ be the point of intersection of its diagonals. Put $\varphi=\angle A O B$. We denote $A B=a, B C=b$, $C D=c, D A=d, B D=m, A C=n$, and $\angle A O B=\varphi$. Let $\mathcal{E}_{1}, \mathcal{E}_{2}, \mathcal{E}_{3}, \mathcal{E}_{4}$ be the areas of the triangles $O A B, O B C, O C D, O D A$, respectively, and let $\mathcal{E}=\mathcal{E}_{1}+\mathcal{E}_{2}+\mathcal{E}_{3}+\mathcal{E}_{4}$. Then

$$
\frac{a+c+m+n}{2 \pi-\left(\mathcal{E}_{1}+\mathcal{E}_{3}\right)+2 \varphi}<\frac{a+b+c+d}{2 \pi-\mathcal{E}} .
$$

Proof. Let $\varphi^{\prime}$ be the angle of the planar triangle with sides $a, A O$, and $B O$, opposite to the side $a$. Then

$$
\frac{a}{A O+B O}>\frac{\varphi^{\prime}}{\pi}>\frac{\varphi-\mathcal{E}_{1} / 2}{\pi}>\frac{\varphi-\left(\mathcal{E}_{1}+\mathcal{E}_{3}\right) / 2}{\pi}
$$

(the first inequality follows from the proof of Lemma 4 , the second is Lemma $1 s$ ). Similarly,

$$
\frac{c}{C O+D O}>\frac{\varphi-\left(\mathcal{E}_{1}+\mathcal{E}_{3}\right) / 2}{\pi}
$$

Estimates $\left(7_{s}\right)$ and $\left(8_{s}\right)$ imply that

$$
x:=\frac{a+c}{m+n}>\frac{\varphi-\left(\mathcal{E}_{1}+\mathcal{E}_{3}\right) / 2}{\pi} .
$$

Similarly,

$$
y:=\frac{b+d}{m+n}>\frac{\pi-\varphi-\left(\mathcal{E}_{2}+\mathcal{E}_{4}\right) / 2}{\pi} .
$$

We substitute these lower bounds for $x$ and $y$ to the identity

$$
z:=\frac{a+c+m+n}{a+c+b+d}=\frac{x+1}{x+y}=1+\frac{1-y}{x+y} .
$$

Since $y<1$, we get an upper bound for $z$. This gives $\left(6_{s}\right)$.

Now we briefly explain the plan of the proof of Theorem $\mathcal{S}^{\prime}$.

The arguments used in cases a) and c) of $\S 4$ can be transferred with minor changes (the natural changes arising due to the spherical excess $\mathcal{E}$ only make the corresponding inequalities better).

In case b), we choose an index $i$ so that an angle vertical to $\angle A_{i-1} A_{i+1} A_{i}$ has the smallest area in the hemisphere. The angle vertical to $\angle A_{i} A_{i+2} A_{i+1}$ is contained in the angle vertical to $\angle A_{i-1} A_{i+1} A_{i}$ (in the hemisphere), because there are no conjugate points on a hemisphere. This leads to a contradiction.

The arguments of $\S 5$ are modified in the same way as those in case c) of $\S 4$. 
Deduction of Theorem $\mathcal{S}$ from Theorem $\mathcal{S}^{\prime}$. First, we note that the curve $\Gamma$ splits into a finite number of parts without self-intersections. Indeed, if a part of $\Gamma$ with selfintersections has sufficiently small length, then its rotation is at least $\pi / 2$. Hence, the number of such parts cannot be infinite.

Let $0=t_{1}<t_{2}<\cdots<t_{n}<t_{n+1}=L(\Gamma)$ be the nodes of such a partition (we may assume that $\Gamma$ is parametrized naturally with the starting point at a node). Set $A_{i}:=\Gamma\left(t_{i}\right), \Gamma_{i}:=\Gamma_{\left[t_{i}, t_{i+1}\right]}$. By $[7$, Theorem 1], for $i=1, \ldots, n$ there exists a sequence of polygonal lines $g_{k}^{i}:\left[t_{i}, t_{i+1}\right] \rightarrow S^{2}(k=1,2, \ldots)$ such that $g_{k}^{i}\left(t_{i}\right)=A_{i}, g_{k}^{i}\left(t_{i+1}\right)=A_{i+1}$, $g_{k}^{i}$ converges to $\Gamma_{i}$ from the right, and $\lim \sup V\left(g_{k}^{i}\right) \leq V\left(\Gamma_{i}\right)$. Moreover, the directions of $g_{k}^{i}$ at the points $A_{i}$ and $A_{i+1}$ converge to the directions (right and left, respectively) of the curve $\Gamma$ at these points.

Let $g_{k}$ denote the union of the polygonal lines $g_{k}^{i}$ over $i=1, \ldots, n$, and let $G_{k}$ be the boundary of the convex hull of $g_{k}$. Then $\lim \sup V\left(g_{k}\right) \leq V(\Gamma)$. Also, $L(\Gamma) \leq$ $\lim \inf L\left(g_{k}\right)$. Finally, since $g_{k} \rightarrow \Gamma$ uniformly, we have $G_{k} \rightarrow \Gamma_{1}$, whence $L\left(G_{k}\right) \rightarrow L\left(\Gamma_{1}\right)$ and $T\left(G_{k}\right) \rightarrow T\left(\Gamma_{1}\right)$. By Theorem $\mathcal{S}^{\prime}$,

$$
T(\Gamma) \geq \lim \sup T\left(g_{k}\right) \geq \lim \sup T\left(G_{k}\right)=T\left(\Gamma_{1}\right) .
$$

This completes the proof.

Unfortunately, we are not able to transfer statement 2 of Theorem $\mathcal{P}$ to the spherical case.

Observe that on the Lobachevsky plane the DNA inequality fails. To see this, consider a triangle $A B C$ in the Lobachevsky plane (with the curvature equal to -1 ) and a polygonal line $\Gamma=A B C C_{1} B_{1} B C A$ with some points $B_{1} \in A B, C_{1} \in A C$. Then $\Gamma_{1}=A B C A$, and

$$
\frac{V(\Gamma)}{V\left(\Gamma_{1}\right)}=2-\frac{S\left(A B_{1} C_{1}\right)}{V\left(\Gamma_{1}\right)}<2-\frac{S\left(A B_{1} C_{1}\right)}{3 \pi} .
$$

Moving the vertex $B$ sufficiently far along the ray $A B_{1}$, we make the ratio $L(\Gamma) / L\left(\Gamma_{1}\right)$ arbitrarily close to 2 , which gives $T(\Gamma)<T\left(\Gamma_{1}\right)$.

We are grateful to V. A. Zalgaller, who drew our attention to the references [3] and [7]. We also thank S. V. Duzhin for his attention.

\section{REFERENCES}

[1] S. Tabachnikov. The tale of a geometric inequality, MASS colloquium lecture, 2001.

[2] J. Lagarias, T. Richardson. Convexity and the average curvature of the plane curves, Geom. Dedicata, 67 (1997), 1-38. MR1468858 (98f:52007)

[3] A.D. Aleksandrov. Intrinsic Geometry of Convex Surfaces. OGIZ, Moscow-Leningrad, 1948; English transl., A.D. Alexandrov. Selected works: Intrinsic Geometry of Convex Surfaces. CRC, 2005. MR0029518 (10:619c)

[4] http://mathworld.wolfram.com/SphericalTrigonometry.html

[5] http://mathworld.wolfram.com/Triangle.html

[6] http://mathworld.wolfram.com/SphericalExcess.html

[7] V. A. Zalgaller. On curves with curvature of bounded variation on a convex surface, Mat. Sbornik, 26(68) (1950), 205-214. (Russian) MR0035043 (11:681a)

Department of Mathematics and Mechanics, St. Petersburg State University, UniverSitetskil Pr. 28, Stary I Peterhof, St. Petersburg 198504, Russia

E-mail address: an@AN4751.spb.edu

St. Petersburg Branch, Steklov Mathematical Institute, Russian Academy of Sciences, Fontanka 27, St. Petersburg 191023, Russia

E-mail address: fedorpetrov@mail.ru

Received 1/AUG/2006 